\title{
The relevance of age at first alcohol and nicotine use for initiation of cannabis use and progression to cannabis use disorders
}

\author{
Silke Behrendt ${ }^{\text {a,* }}$, Katja Beesdo-Baum ${ }^{\text {a }}$, Michael Höfler ${ }^{\text {a }}$, Axel Perkonigg ${ }^{\mathrm{e}}$, Gerhard \\ Bühringer ${ }^{\mathrm{a}, \mathrm{d}}$, Roselind Lieb ${ }^{\mathrm{b}, \mathrm{c}}$, Hans-Ulrich Wittchen ${ }^{\mathrm{a}, \mathrm{c}}$ \\ a Institute of Clinical Psychology and Psychotherapy, Technische Universitaet Dresden, Chemnitzer Str. 46, D- \\ 01187 Dresden, Germany \\ b University of Basel, Department of Psychology, Clinical Psychology and Epidemiology, Missionsstraße 60-62, \\ 4055 Basel, Switzerland \\ c Max Planck Institute of Psychiatry, Kraepelinstr. 2-10, D-80804 Munich, Germany \\ d IFT Institut für Therapieforschung, Parzivalstraße 25, D-80804 Munich, Germany \\ e Institute of Applied Research, Protestant University of Applied Sciences, Paulusweg 6, 71638 Ludwigsburg, \\ Germany
}

\begin{abstract}
Background: A younger age at onset of use of a specific substance is a well-documented riskfactor for a substance use disorder (SUD) related to that specific substance. However, the cross-substance relationship between a younger age at onset of alcohol use (AU) and nicotine use (NU) and the risk of cannabis use disorders (CUD) in adolescence and early adulthood remains unclear.

Aims: To identify the sequence of and latency between initial AU/NU and initial cannabis use (CU). To investigate whether younger age at AU- and NU-onset is associated with any and earlier CU-onset and a higher risk of transition from first CU to CUD, taking into account externalizing disorders (ED) and parental substance use disorders as putative influential factors. Methods: Prospective-longitudinal community study with $\mathrm{N}=3021$ subjects (baseline age 14-24) and up to four assessment waves over up to ten years with additional direct parental and family history information. Substance use and CUD were assessed with the DSM-IV/M-CIDI.

Results: Most subjects with CU reported AU (99\%) and NU (94\%). Among users of both substances, 93\% reported AU prior to CU (87\% for NU). After adjustment for ED and parental substance use disorders younger age at AU-onset was associated with any CU. Younger age at NU-onset was associated with earlier CU initiation. Younger age at AU- and NU-onset was not associated with a higher risk of CUD.

Conclusions: The cross-substance relevance of younger age at first AU and NU for the risk of CUD is limited to early CU involvement.
\end{abstract}

Keywords: Adolescence Epidemiology DSM-IV-cannabis use disorders Etiology Parental disorder Family history 


\section{Introduction}

A younger age at onset of use of a specific substance is a well-documented risk factor for substance use disorders (SUD, DSM-IV-substance abuse or dependence) related to this specific substance (Behrendt et al., 2009; Breslau et al., 1993; Buchmann et al., 2009; Chen et al., 2005; Dawson et al., 2008; DeWit et al., 2000). Nevertheless, there are still restrictions to the understanding of the role of younger age at onset of substance use (SU) in substance use disorder development. It remains unclear whether younger age at substance use onset is a substance-specific risk-factor or a risk factor for substance use disorders across substance classes. It is also unclear whether a cross-substance association between a younger age at substance use onset and substance use disorders is independent of important influential factors that may constitute a general underlying vulnerability for early substance use and SUD. Therefore, we investigate the cross-substance relationship between younger age at onset of use of one substance for the risk of substance use disorders related to another substance. Here, we focus on the role of younger age at onset of alcohol use (AU) and nicotine use (NU) for the risk of cannabis use disorders (CUD). This is due to several reasons: at least experimental $\mathrm{AU}$ and NU are almost normative experiences in adolescence in western societies and most likely the first substance use experiences (Poelen et al., 2005; Wittchen et al., 2008; Young et al., 2002). Epidemiological evidence indicates that adolescents are unusually naïve to AU and NU at first cannabis use (CU) (Bonomo et al., 2004; Everett et al., 1999; Hayatbakhsh et al., 2007; Kandel and Yamaguchi, 1993; Monshouwer et al., 2005; Palmer et al., 2009; Patton et al., 2002; Poelen et al., 2005; Wagner and Anthony, 2002a; Wittchen et al., 2008; Young et al., 2002). Cannabis is the most widely used illegal substance in western societies (European Monitoring Centre for Drugs and Drug Addiction, 2010; United Nations Office on Drugs and Crime, 2010). For a proportion of users CU leads to CUD, considerable psychosocial sequelae and treatment need (Coffey et al., 2002; European Monitoring Centre for Drugs and Drug Addiction, 2010; Georgiades and Boyle, 2007; Hayatbakhsh et al., 2007; Patton et al., 2002). Investigating the relationship between younger age at onset of AU and NU and the risk of CUD is important for early identification of youth at risk of CUD and for the planning of timely preventive efforts. For this matter, it is also important to investigate the latency between first AU/NU and first CU-involvement.

To date, the specific relationship between a younger age at first AU and NU and CUD risk remains unclear, despite well-documented general cross-substance associations for any substance use and substance use disorders (Grucza and Bierut, 2006; Lynskey et al., 2003; Palmer et al., 2009). Such relationships may exist because any AU and NU as well as younger age at AU- and NU-onset are associated with the risk of at least experimental CU and younger age at CU-onset (Caris et al., 2009; Kokkevi et al., 2006; Sartor et al., 2009; von Sydow et al., 2002; Wagner and Anthony, 2002b), the latter of which predicts CUD (Chen et al., 2005).

It also remains unclear whether primacy of AU or NU (i.e., which of the two substances was tried first) which may indicate a deviant substance use initiation pattern and thus CUD risk and prevention need, plays a role in this regard (Degenhardt et al., 2009). Importantly, other factors may also play a role in the relationship between younger age at first AU and NU and the risk of CUD. It has been proposed that early use of different substances and different substance use disorders share underlying influential factors (Morral et al., 2002; Palmer et al., 2009; Sartor et al., 2009), most importantly externalizing disorders, genetic factors, and familial SUD. Externalizing disorders and parental substance use disorders are related to offspring substance use disorders and younger age at substance use onset (Elkins et al., 2007; King et al., 2004; Lieb et al., 2001, 2002; McGue and Iacono, 2008; Obot et al., 2001). However, younger age at first AU is a risk factor for alcohol use disorders independent of 
these factors (Dawson et al., 2008), underlining the possible specific importance of younger age at substance use onset (Agrawal et al., 2009). As shown for alcohol, neurodegenerative effects of early substance use may lead to reduced behavioral control (Nixon and McClain, 2010), a factor relevant for all kinds of substance use disorders including CUD (Elkins et al., 2007; Hayatbakhsh et al., 2008). Therefore, we hypothesize to find a cross-substance relationship between younger age at AU- and NU-onset and an elevated CUD risk independent of younger age at CU-onset, externalizing disorders and parental SUD. Considering these factors can help understanding the role of early AU/NU in CUD etiology and identifying the most important indicators of CUD risk.

We investigate in a community sample of adolescents and young adults

(1) the sequence of and latency between AU/NU and CU initiation,

(2) whether a younger age at AU-onset and younger age at NUonset are associated with a higher risk of any $\mathrm{CU}$, younger age at first CU, and CUD,

(3) whether the association between a younger age at AU-onset and younger age at NU-onset and the risk of any $\mathrm{CU}$ and younger age at first $\mathrm{CU}$ is independent of externalizing disorders, parental alcohol dependence and parental illegal substance dependence; whether the association between a younger age at AU-onset and younger age at NU-onset and the risk of CUD is independent of externalizing disorders, parental alcohol and illegal substance dependence and younger age at CU-onset,

(4) whether results for (2) and (3) differ by primacy of AU or NU.

\section{Methods}

\subsection{Study sample and design}

The prospective-longitudinal EDSP (Early Developmental Stages of Psychopathology) study investigates vulnerabilities and risk-factors of substance use and disorders in a community sample of 3021 adolescents and young adults aged 14-24 at study baseline (T0). The study includes three follow-up assessments (T1, T2, T3). Detailed information on the study has been provided (Beesdo et al., 2007; Lieb et al., 2000; Wittchen et al., 1998b). The baseline sample was randomly drawn from government registries in metropolitan Munich, Germany in 1994. To emphasize early developmental stages, subjects aged 14-15 years were sampled at twice the probability and subjects aged 22-24 were sampled at half the probability of those aged 16-21 years. Follow-up examinations were carried out approximately 1.6 years (T1, median interval since baseline), 3.5 years (T2) and 8.2 years (T3) after T0. At T1, only subjects aged $14-17$ years at T0 were assessed $(\mathrm{N}=1228$; response rate $88.0 \%) .84 .3 \%(\mathrm{~N}=$ $2548)$ of $\mathrm{T} 0$ participants completed $\mathrm{T} 2,73.2 \%(\mathrm{~N}=2210) \mathrm{T} 3$. The T3-age range was $21-34$ years.

\subsection{Diagnostic assessment}

At all waves, participants were interviewed with the computer-assisted standardized MunichComposite International Diagnostic Interview (DIA-X/M-CIDI) (Wittchen et al., 1998a; Wittchen and Pfister, 1997), an updated version of the World Health Organisation (WHO) CIDI (Wittchen and Semmler, 1990) (lifetime version at T0; interval version at T1-T3). For DSM-IV diagnoses, computerized M-CIDI/DSM-IV algorithms were applied. To help respondents, e.g., in answering symptom questions, the DIA-X/M-CIDI includes a respondent's booklet with symptom lists and cognitive aids. Test-retest reliability and validity 
of DIA-X/M-CIDI diagnoses have been established (Lachner et al., 1998; Reed et al., 1998; Wittchen et al., 1998a). Externalizing disorder diagnosis was derived from T1 parental reports on conduct disorder and T2 participants' reports on conduct and antisocial personality disorder resulting in externalizing disorder-information on 2638 subjects.

2.2.1. Assessment of substance use and disorders. Substance use and disorders were assessed with the three DIA-X/M-CIDI-sections on alcohol, nicotine, and medication and illegal substances (Wittchen et al., 2008). For CUD assessment minimal CU ( $>4$ times) was required. $\mathrm{N}=142$ subjects who declined answering questions on illegal substances truthfully at one or more wave(s) were excluded from all analyses. Substance use (disorder) categories considered here were: any AU, NU and CU, and DSM-IV-CUD (cannabis abuse and dependence).

Baseline CU in the younger cohort (OR 0.66, 95\% CI: 0.4-0.9, $\mathrm{p}=0.015$ ) and baseline NU in the older cohort (OR 0.71, 95\% CI: $0.5-0.9, \mathrm{p}=0.027$ ) predicted attrition at T3.

2.2.2. Parental diagnoses. The EDSP includes a family supplement (Lieb et al., 2000). Parents were directly interviewed at T1 and T3 (1056 T1 parent-interviews). Since the primary objective of T3 was to investigate first-degree relatives of offspring with a lifetime affective disorder, only these relatives were invited (252 subjects with T3 parent-interviews). Directly interviewed parents underwent assessment with the DIA-X/M-CIDI. Attempts were made to ensure interviewer blindness to offspring diagnostic status.

Family history $(\mathrm{FH})$ information on mental disorders in both parents was obtained from offspring (T0, T2, T3). FH-component items were designed using a modified version of the Family History- Research Diagnostic Criteria as a model (Merikangas et al., 1998). At T0, DIA-X/M-CIDI stem questions and questions to determine whether the relative sought professional help because of the symptoms were used. At T2 and T3, a FH-module with fully structured sections covering DSM-IV criteria was applied.

Here, parental diagnoses were determined as follows: whenever available, direct information from $\mathrm{T} 1 \mathrm{and} /$ or $\mathrm{T} 3(\mathrm{~N}=1152$ mothers, $\mathrm{N}=211$ fathers, $\mathrm{N}=1189$ any parent) was used. If this was impossible, T3 FH-information was used. T2 FH-information was used when direct information and $\mathrm{T} 3 \mathrm{FH}$-information was unavailable. T0 FH-information was used when all other information types were unavailable. This algorithm is based on agreement patterns between FH and direct interviews (highest agreement at T3). Values were comparable with previous FH-reporting (Vandeleur et al., 2008). Direct or FH-information on parental substance dependence is available for $\mathrm{N}=2720$ fathers and $\mathrm{N}=2763$ mothers of the $\mathrm{N}=$ 3021 adolescent and young adult subjects (T0 FH-information did not permit differentiation between substance abuse and dependence and was not used).

\subsection{Statistical analysis}

To ensure community representativeness, data were weighted to account for different sampling probabilities at baseline according to age, and response rates at baseline varying over age, gender, and geographic region. The Stata Software package 11.0 (StataCorp., 2009) was used for all calculations and to compute robust confidence intervals, and p-values (by applying the Huber-White sandwich matrix) required when basing analyses on weighted data (Royall, 1986). Cumulative lifetime status was used in all analyses and created by defining caseness as having reported the respective items at any assessment (covering the lifetime 
history up to the age at last assessment). This permitted using information from subjects who dropped out of the study during follow-up.

\subsubsection{Sequence of and latency between AU/NU and CU initiation. The Kaplan-Meier} estimator (Therneau and Grambsch, 2000) was used to estimate age-dependent cumulative lifetime incidence and timedependent survival rates of substance use.

2.3.2. Risk of any and earlier CU. To investigate associations between covariates of interest (younger age at onset of AU and NU (continuous variable, age in years, older age at onset compared to younger age at onset), externalizing disorders, parental alcohol or illegal substance dependence) and the risk of any and earlier CU, we used logistic and linear regression analyses ("crude model"). Multiple logistic and linear regression analyses were conducted with all covariates of interest ("multiple model"). Both the "crude" and the "multiple" model were additionally adjusted for maximum age as observed until the last assessment and gender. Regressions including parental substance dependence were additionally adjusted for parental age and information level (three dummy variables for four different sources of information about parents; see Section 2.2.2).

\subsubsection{Risk of CUD. Cox regressions were applied to assess overall differences in risk of} transition from first CU to CUD (time scale = years from first CU to CUD) over time as a function of the covariates of interest listed above and age at CU onset. Different curves before assessing differences according to covariates were allowed for according to year of birth and gender ("stratified Cox regression") (Therneau and Grambsch, 2000). Multiple Cox regression analyses were conducted with all covariates of interest and age of CU-onset.

The proportional hazard assumption was tested using Schoenfeld residuals (Therneau and Grambsch, 2000). If the assumption was violated, to improve the model fit and to assess how strongly the hazard ratios depended on time the interaction term covariate $\times$ [number of years since first CU] was added to the model. Here, the model-based time-dependent hazard ratio equals HR (main effect of covariate) $\times$ HR (interaction effect of covariate) ${ }^{\text {number of years }}$. After the inclusion of the interaction term the proportional hazard assumption was tested again (in the Cox regression as implemented in Stata, cases with CU and CUD onset within the same year are excluded. To prevent this, we shifted the time scale for the analysis 1 year upwards, replacing 0 years by 1 year, 1 year by 2 years, and so on and afterwards shifted the results back by one year.).

For the Cox regression, we used data from subjects with information on age of onset of lifetime $\mathrm{CU}(\mathrm{N}=1343)$ who were willing to answer drug questions truthfully.

We repeated all analyses with a stricter definition of early substance use experiences. Here, we included only cases with AU before NU or no NU (AU-First), respectively only cases with NU before AU or no AU (NU-First; AU-First and NU-First refer to mutually exclusive cases. Therefore separate multiple models were run for the AU-First and NU-First analyses with age of onset of any AU (respectively any NU), externalizing disorders, parental alcohol dependence and illegal substance dependence and age at first CU (the latter only in Cox regression analysis) as additional covariates.).

Paternal and maternal substance use disorders are considered separately. Because of limited power a variable for parental (i.e., paternal and/or maternal) illegal substance dependence was used in some analyses, and parental illegal substance dependence was excluded from AUFirst and NU-First multiple logistic regression models. 


\section{Results}

\subsection{Sequence of and latency between $A U / N U$ and $C U$ initiation}

As reported previously (Wittchen et al., 2008), the cumulative incidence rate up to T3 was $97.7 \%$ for $\mathrm{AU}, 79.2 \%$ for $\mathrm{NU}$ and $50.7 \%$ for CU. The main incidence periods (i.e., the steepest increase in the Kaplan-Meier estimates for cumulative lifetime incidence) were ages 10-16 (AU), 11-17 (NU) and 14-19 (CU).

Almost all subjects with lifetime CU reported lifetime AU (99.6\%) and NU (94.7\%).

Most (93.0\%) users of alcohol and cannabis reported first AU as prior to CU. 4.4\% reported AU and CU initiation in the same year. 2.5\% reported AU as secondary. A comparable pattern was found for users of nicotine and cannabis: $87.5 \%$ reported primary NU, $8.8 \%$ initiation in the same year and $3.7 \% \mathrm{NU}$ as secondary.

In cases in which CU occurred after AU or in the same year, $10 \%$ of all transitions from first AU to first CU had occurred at one year after first AU (Fig. 1), 20-30\% at two years and about $40 \%$ at three years. The respective proportions were $10-20 \%, 30-40 \%$ and $50 \%$ for transitions from first NU to first CU.

\subsection{The risk of any and early $C U$ and $C U D$}

Younger age at AU-onset (OR: 0.89) and NU-onset (OR: 0.91), paternal (OR: 1.71) and maternal alcohol dependence (OR: 1.62) and externalizing disorders (OR: 3.31) were associated with a higher risk of any CU (logistic regression results are shown in Table 1).

Younger age at AU- and NU-onset were associated with an average 0.17 (respectively 0.30) years younger age at $\mathrm{CU}$-onset. Externalizing disorders and paternal alcohol dependence were also associated with earlier CU-onset (linear regression results are shown in Table 2).

Younger age at AU-onset, paternal alcohol dependence and externalizing disorders were associated with a higher risk of CUD (Cox regression results are shown in Table 3).

Externalizing disorders were marginally associated with a lower speed of transition to CUD (hazard ratio (HR) main effect: 1.47; HR interaction effect: 1.12; 95\% CI: 1.001-1.26; $\mathrm{p}=$ 0.047).

\subsection{Multiple models}

In the multiple models, associations with a higher risk of any CU remained significant for younger age at AU-onset, externalizing disorders and paternal alcohol dependence (Table 1). Younger age at NU-onset, externalizing disorders and paternal alcohol dependence remained significant factors for earlier CU-onset (Table 2). Younger age at CU-onset (HR: 0.77), externalizing disorders (HR: 1.69) and paternal alcohol dependence (HR: 1.47) were associated with a higher CUD-risk. Externalizing disorders were associated with a slower transition to CUD (HR main effect: 1.14; HR interaction effect: 1.17 ; 95\% CI: $1.03-1.33$; $\mathrm{p}=$ 0.013) (Table 3).

\subsection{AU-First and NU-First}


Risk of CU: Younger age at onset of AU-First was associated with a higher risk of CU (OR: 0.82). In the AU-First multiple model, younger age at onset of AU-First, externalizing disorders, paternal and maternal alcohol dependence were associated with a higher CU risk as were younger age at AU-onset and externalizing disorders in the NU-First multiple model (Table 1).

Earlier CU-onset: Younger age at onset of AU-First and NU-First were associated with an average $0.26(0.14)$ years younger age of CU-onset, but only in the crude model (Table 2).

Risk of CUD: Younger age at AU-First onset was associated with a higher CUD risk (HR: 0.88). In the AU-First multiple model only younger age at CU-onset was associated with a higher risk of CUD. In the NU-First multiple model younger age at CU-onset and maternal alcohol dependence were associated with higher CUD risk (Table 3).

\section{Discussion}

We investigated the cross-substance relationship between, respectively a younger age at onset of alcohol use and a younger age at onset of nicotine use and the risk of cannabis use disorders, taking into account age of cannabis use onset, externalizing disorders, and parental substance use disorders as important influential factors for the risk of cannabis use and disorders. The main findings are: (1) Almost all cannabis users had prior experience with alcohol (99.6\%) and nicotine (94.7\%) use. Cannabis use almost invariably occurred after first alcohol and first nicotine use. (2) After adjustment for externalizing disorders and parental substance dependence, a younger age at first alcohol use was associated with a higher risk of any cannabis use; a younger age at first nicotine use with a younger age at first cannabis use. (3) Younger age at first alcohol and nicotine use was not associated with the risk of cannabis use disorders after adjustment for externalizing disorders, early onset of cannabis use and parental substance dependence. (4) Overall, taking into account the temporal order of onset of alcohol and nicotine use did not produce more consistent associations with the risk of cannabis use and disorders.

In western societies, substance use almost normatively begins with alcohol or nicotine use. Typically, the main incidence phases for alcohol and nicotine use occur earlier than the main incidence phase for cannabis use (Degenhardt et al., 2009; Kandel and Yamaguchi, 1993; Wagner and Anthony, 2002a; Wittchen et al., 2008; Young et al., 2002). Our findings agree with this evidence. However, here and in other studies (Degenhardt et al., 2009), the sequence pattern for nicotine and cannabis was less distinct than for alcohol and cannabis (e.g., larger proportion of cannabis use initiation before nicotine use). Still, the sequence-pattern suggests that alcohol and nicotine use may play a role for cannabis effects and for developing cannabis use disorders for example via cross-tolerance or cross-sensitivity (Perkins et al., 2009). Here, $40-50 \%$ of cannabis use-onsets occurred within three years after initial alcohol/nicotine use. For this considerable proportion of subjects, the time-window for efforts to prevent multiple substance use (Pape et al., 2009) and its potential sequelae is relatively narrow.

To our knowledge it is, to date, unclear whether a cross-substance relationship exists between younger age at first alcohol and nicotine use and the risk of cannabis use disorders and whether this relationship is independent of important factors as externalizing disorders, early cannabis use onset and parental substance dependence. Our study adds to current knowledge by showing that with few exceptions, younger age at first alcohol use and at first nicotine use were associated with the risk of (early) cannabis use and cannabis use disorders. However, 
importantly, most of these cross-substance associations were attenuated after adjustment for externalizing disorders and parental substance dependence. In the associations that remained significant, specific factors related to younger age at first alcohol or nicotine use may be influential as discussed below. However, these factors may only influence cannabis use; we found no evidence for a role of younger age at first alcohol or nicotine use in the risk of cannabis use disorders after adjustment for the abovementioned factors.

\subsection{Risk of any cannabis use}

Here, a younger age at first alcohol use was associated with an increased risk of cannabis use, independent of externalizing disorders and parental substance dependence. A younger age at first nicotine use was also associated with a higher risk of cannabis use, but not after adjustment for externalizing disorders and parental substance dependence. In conclusion, early alcohol use initiation is a risk factor not only for alcohol dependence (DeWit et al., 2000; Ellickson et al., 2004; Sartor et al., 2009) but also for cannabis use. A specific factor in this relationship may be that a younger age at first alcohol use is associated with heavy drinking (Buchmann et al., 2009). One may speculate that subjects with heavy drinking experience may intend to experience intoxication related to another substance and therefore try cannabis.

\subsection{Risk of younger age at cannabis use onset}

We extend earlier findings (Kokkevi et al., 2006) by showing that a younger age at first alcohol use and a younger age at first nicotine use were associated with a younger age at first cannabis use. For younger age at first nicotine use the association was independent of externalizing disorders and parental substance dependence, suggesting that younger age at onset of nicotine use is a risk factor for earlier cannabis use initiation. Thus, importantly, while no direct association between earlier onset of nicotine use and cannabis use disorders was found, earlier nicotine use may indeed be of indirect relevance for cannabis use disorder risk via earlier onset of cannabis use, which is a risk factor for cannabis use disorders (Chen et al., 2005). Still, it has to be noted that the average difference in age at first cannabis use by earlier smoking onset ( 0.2 years) found here may be too small to play a role in cannabis use disorder development. Younger age at first alcohol use was not associated with a younger age at first cannabis use after adjustment for parental alcohol dependence, for example. Paternal alcohol dependence is related to younger age at onset of both alcohol and cannabis use (Obot et al., 2001). Also, a considerable overlap of genetic factors exists for age at first alcohol and age at first cannabis use. This overlap is smaller for nicotine and cannabis (Sartor et al., 2009).

The differences in findings for younger age at alcohol and nicotine use may indicate that in addition to a general vulnerability for early substance use (McGue and Iacono, 2008), specific risk factors may be important for early onset of use of different substances.

\subsection{Risk of cannabis use disorders}

Our study adds to current knowledge by showing that a younger age at first alcohol use is associated with a higher risk of cannabis use disorders. However, against our expectation, this association was non-significant after adjustment for important covariates (externalizing disorders, early cannabis use onset and parental substance dependence). Thus, the relevance of a younger age at first alcohol and nicotine use was limited to any cannabis use, respectively especially early cannabis use initiation. In contrast, younger age at first cannabis use, paternal 
alcohol dependence and externalizing disorders were consistently associated with cannabis use and disorders. This probably confirms the importance of these factors as more general risk factors for early substance use and substance use disorders (Elkins et al., 2007; Hayatbakhsh et al., 2008; King et al., 2004; Obot et al., 2001). The findings on younger age at first cannabis use may underline the importance of substance-specific associations between younger age at first use and the risk of a substance use disorder (Agrawal et al., 2009; Buchmann et al., 2009; Dawson et al., 2008). Differential sensitivity to cannabis effects in adolescence may be of importance in this relationship for cannabis (Schramm-Sapyta et al., 2009). For example, animal studies suggest less acute aversive effects of cannabis in adolescence (Quinn et al., 2008; Schramm-Sapyta et al., 2007) as well as impairment of working memory and (non-) spatial learning after cannabis exposure (Cha et al., 2006; O'Shea et al., 2004; Quinn et al., 2008). Cannabis exposure in adolescence may also alter the development of the endocannabinoid system (Malone et al., 2010). In early cannabis users, these factors may contribute to the more intense cannabis use necessary for cannabis use disorder development.

While certain sequences of substance use initiation can be related to dependence risk (Degenhardt et al., 2009) our results do not suggest that the sequence of alcohol and nicotine use initiation plays a role in the risk of cannabis use and disorders. The primacy of alcohol use with respect to nicotine use may not represent a deviant initiation pattern associated with further problematic development.

In conclusion, our results do not suggest a direct cross-substance momentum of younger age at first alcohol and nicotine use for the risk of cannabis use disorders but underline the importance of younger age at first cannabis use as a substance-specific factor, externalizing disorders and paternal alcohol dependence in this regard. Still, importantly, younger age at first nicotine use may be of indirect relevance for the risk of cannabis use disorders. Also, younger age at first alcohol use was related to any cannabis use independent of externalizing disorders and familial substance dependence. Delaying first alcohol use may serve to prevent any cannabis use while delaying first nicotine use may serve to prevent especially early cannabis use and thus even help reducing the risk of cannabis use disorders.

\subsection{Limitations}

We used age of onset information that may underlie recall bias. Aggregation of data over the assessment waves was necessary because of restricted power. Therefore, the temporal order for covariates and outcomes was not established. However, the sequence of initiation shows that initial cannabis use mostly occurs after initial alcohol and nicotine use. Parental diagnoses were partially based on FH-information. However, direct information was used whenever possible. Also, agreement between $\mathrm{FH}$ and direct information on alcohol dependence is at least fair (Vandeleur et al., 2008).

\subsection{Future research}

As most cannabis users also use alcohol and nicotine, mostly prior to cannabis, future research should investigate crosstolerance and -sensitivity for these three substances in adolescents to further clarify the role of alcohol and nicotine use for cannabis use development. To further improve knowledge on the role of early substance use as a risk factor for substance use disorders, the limitation of the role of younger age at first alcohol and nicotine use to early stages of cannabis involvement should be investigated. 


\section{Role of funding source}

This paper was prepared in the context of the project 'Community-based need evaluation II and allocation and transfer' (primary investigator: H.-U. Wittchen) of the German Addiction Research Network ASAT (Allocating Substance Abuse Treatments to Patient Heterogeneity). This work is a further part of the Early Developmental Stages of Psychopathology (EDSP) Study and is funded by the German Federal Ministry of Education and Research (BMBF) project nos. 01EB9405/6, 01EB9901/6, EB01016200, 01EB0140 and 01EB0440. Parts of the fieldwork and analyses were supported additionally by grants from the Deutsche Forschungsgemeinschaft (DFG) LA1148/1-1, WI2246/1-1, WI709/7-1 and WI709/8-1.

\section{Contributors}

Dr. Silke Behrendt planned the analysis and wrote the manuscript.

Dr. Buehringer, Dr. Beesdo-Baum, Dr. Perkonigg, Dr. Wittchen, Dr. Lieb and Dr. Höfler provided supervision of and substantial contribution to the writing of the manuscript. Dr. Höfler also provided supervision and substantial contribution to the planning and conduction of the statistical analysis.

All authors contributed to and have approved of the final version of the manuscript.

\section{Conflict of interest}

Gerhard Bühringer: Alcohol, pharmaceutical and tobacco industry: none. Gaming industry: research has been funded by the Bavarian State Ministry of the Environment and Public Health (State gambling monopoly) and organisations of the gaming industry (private gambling).

Dr. Hans-Ulrich Wittchen has no connection with the alcohol and tobacco industry. Dr. Wittchen is and has been a member of advisory boards of several pharmaceutical companies. He has received travel reimbursements and research grant support from: Essex Pharma, Sanofi, Pfizer, Organon, Servier, Novartis.

All other authors: No conflict of interest.

\section{Acknowledgements}

The principal investigators of the EDSP study are Dr. Hans-Ulrich Wittchen and Dr. Roselind Lieb. Core staff members of the EDSP group are: Dr. Kirsten von Sydow, Dr. Gabriele Lachner, Dr. Axel Perkonigg, Dr. Peter Schuster, Dr. Michael Höfler, Dipl.-Psych. Holger Sonntag, Dr. Tanja Brückl, Dipl.-Psych. Elzbieta Garczynski, Dr. Barbara Isensee, Dr. Agnes Nocon, Dr. Chris Nelson, Dipl.-Inf. Hildegard Pfister, Dr. Victoria Reed, Dipl.-Soz. Barbara Spiegel, Dr. Andrea Schreier, Dr. Ursula Wunderlich, Dr. Petra Zimmermann, Dr. Katja Beesdo-Baum, Dr. Antje Bittner, Dr. Silke Behrendt and Dr. Susanne Knappe. Scientific advisers are Dr. Jules Angst (Zurich), Dr. Jürgen Margraf (Basel), Dr. Günther Esser (Potsdam), Dr. Kathleen Merikangas (NIMH, Bethesda), Dr. Ron Kessler (Harvard, Boston) and Dr. Jim van Os (Maastricht). The EDSP project and its family genetic supplement has been approved by the Ethics Committee of the Medical Faculty of the Technische Universitaet Dresden (no: EK-13811). All participants provided informed consent. 


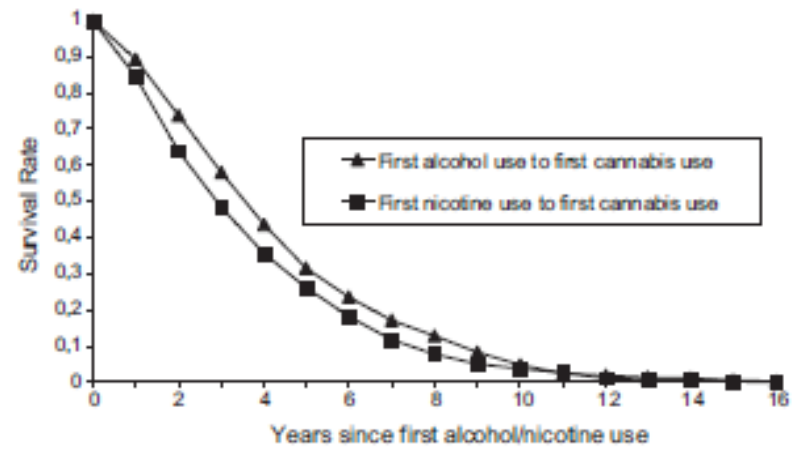

Fig. 1. Latency from first alcohol or nicotine use to initial cannabis use.

Table 1

Earlier onset of alcohol and nicotine use, externalizing disorders, parental substance dependence and the risk of any cannabis use. ${ }^{d, g}$

\begin{tabular}{|c|c|c|c|}
\hline & \multicolumn{3}{|c|}{ Any cannabis use } \\
\hline & Odds ratio & 95\%Cl & $p$ \\
\hline \multicolumn{4}{|l|}{ Crude model ${ }^{a, b}$} \\
\hline Age at first alcohol use $(N-2790)^{F}$ & 0.89 & $0.8-0.9$ & $<0.001$ \\
\hline Age at first nicotine use $(\mathrm{N}-2201)^{\mathrm{E}}$ & 0.91 & $0.8-0.9$ & $<0.001$ \\
\hline Externalizing disorder $(\mathrm{N}-2512)^{f}$ & 3.31 & $2.4-4.5$ & $<0,001$ \\
\hline Paternal alcohol dependence $(N-2451)$ & 1.71 & $1.2-24$ & 0.002 \\
\hline Maternal alcohol dependence $(\mathrm{N}-2577)$ & 1.62 & $1.0-25$ & 0.024 \\
\hline Parental illegal drug dependence $(\mathrm{N}-2462)$ & 1.13 & $0.5-24$ & 0.739 \\
\hline \multicolumn{4}{|l|}{ Multiple model $(N-1743) a, b$} \\
\hline Age at first alcohol use & 0.93 & $0.8-0.9$ & 0.014 \\
\hline Age at first nicotine use ${ }^{2}$ & 0.95 & $0.9-1.0$ & 0.073 \\
\hline Externalizing disorder $f$ & 2.61 & $1.7-3.9$ & $<0.001$ \\
\hline Paternal alcohol dependence & 1.63 & $1.02-26$ & 0.039 \\
\hline Maternal alcohol dependence & 1.63 & $0.8-3.1$ & 0.128 \\
\hline Parental illegal drug dependence & 0.71 & $0.2-1.8$ & 0.470 \\
\hline \multicolumn{4}{|l|}{ Crude model (AU-First; NU-First)* } \\
\hline AU-First $(N-1636)^{F}$ & 0.82 & $0.7-0.9$ & $<0.001$ \\
\hline NU-First $(N-651)^{\mathrm{E}}$ & 0.95 & $0.8-1.0$ & 0.359 \\
\hline \multicolumn{4}{|l|}{ Multiple model (AU-First; $N-874)^{*}$} \\
\hline AU-First ${ }^{-}$ & 0.90 & $0.8-0.9$ & 0.024 \\
\hline Age at first nicotine use ${ }^{2}$ & 0.97 & $0.8-1.0$ & 0.562 \\
\hline Externalizing disorder ${ }^{f}$ & 3.02 & $1.7-5.3$ & $<0.001$ \\
\hline Paternal alcohol dependence & 2.12 & $1.07-4.2$ & 0.030 \\
\hline Maternal alcohol dependence & 2.90 & $1.2-7.0$ & 0.017 \\
\hline \multicolumn{4}{|l|}{ Multiple model (NU-First; $N-483)^{n}$} \\
\hline NU-First ${ }^{c}$ & 1.07 & $0.9-1.2$ & 0.248 \\
\hline Age at first alcohol use & 0.81 & $0.7-0.9$ & 0.003 \\
\hline Externalizing disorder $f$ & 290 & $1.3-6.4$ & 0.008 \\
\hline Paternal alcohol dependence & 1.24 & $0.5-2.8$ & 0.595 \\
\hline Maternal alcohol dependence & 0.76 & $0.2-2.3$ & 0.636 \\
\hline
\end{tabular}

- Crude model: adjusted for offspring gender and maximum age as observed until the last assessment; multiple model: all covariates of interest entered into one regression model adjusted for offspring gender and age.

All analyses including parental disorders were additionally adjusted for information level and parental age.

c Continuous variable scaled as number of years.

Case numbers in analyses vary depending on the number of subjects with information on age at first alcohol use, age at first nicotine use and parental disorders.

- AU-First: age of onset of AU in cases with AU prior to NU or no NU; NU-First: age of onset of NU in cases with NU prior to AU or no AU; in the AU-First multiple model and the NU-First multiple model, parental illegal drug dependence was not included because of restricted power.

"Conduct disorder, antisocial personality disorder.

$8 \mathrm{~N}-142$ excluded because of unwillingness to answer drug questions truthfully. 


\begin{tabular}{|c|c|c|c|}
\hline & \multicolumn{3}{|l|}{ Age at first cannabis use ${ }^{c}$} \\
\hline & Linear regression coef. & $95 \times \mathrm{Cl}$ & p \\
\hline \multicolumn{4}{|l|}{ Crude modele,b } \\
\hline Age at first alcohol use $(\mathrm{N}-1335)^{\mathrm{E}}$ & 0.17 & $0.1-0.2$ & $<0.001$ \\
\hline Externalizing disorder $(N-1199)^{f}$ & -1.18 & -1.5 to -0.8 & $<0.001$ \\
\hline Paternal alcohol dependence $(\mathrm{N}-1176)$ & -0.90 & -1.4 to -0.3 & 0.002 \\
\hline Maternal alcohol dependence $(N-1226)$ & -0.01 & -0.7 to 0.7 & 0.967 \\
\hline Paternal illegal drug dependence $(N-1176)$ & -0.46 & -2.1 to 1.2 & 0.568 \\
\hline Age at first alcohol use & 0.07 & -0.0 to 0.1 & 0.052 \\
\hline Age at first nicotine use ${ }^{\varepsilon}$ & 0.24 & $0.1-0.3$ & $<0.001$ \\
\hline Externalizing disorder $f$ & -1.03 & -1.4 to -0.6 & $<0.001$ \\
\hline Paternal alcohol dependence & -0.67 & -1.2 to -0.1 & 0.018 \\
\hline Maternal alcohol dependence & -0.02 & -0.6 to 0.6 & 0.932 \\
\hline Paternal illegal drug dependence & -0.23 & -1.7 to 1.3 & 0.75 \\
\hline Maternal illegal drug dependence & 1.83 & -0.0 to 3.7 & 0.059 \\
\hline Age at first nicotine use ${ }^{\varepsilon}$ & 0.37 & $0.2-0.5$ & $<0.001$ \\
\hline Externalizing disorder $f$ & -0.88 & -1.4 to -0.3 & 0.002 \\
\hline Paternal alcohol dependence & -0.83 & -1.5 to -0.1 & 0.019 \\
\hline Maternal alcohol dependence & -0.36 & -0.9 to 0.3 & 0.25 \\
\hline Parental illegal drug dependence & 0.02 & -0.9 to 1.0 & 0.963 \\
\hline \multicolumn{4}{|l|}{ Multiple model (NU-First; $N-293)^{E}$} \\
\hline NU-Firste & -0.06 & -0.2 to 0.1 & 0.44 \\
\hline Age at first alcohol use ${ }^{\varepsilon}$ & 0.27 & $0.07-0.5$ & 0.00 \\
\hline Externalizing disorderf & -1.03 & -1.7 to -0.3 & 0.00 \\
\hline Paternal alcohol dependence & -0.89 & -1.8 to 0.1 & 0.081 \\
\hline Maternal alcohol dependence & 0.24 & -1.1 to 1.7 & 0.737 \\
\hline Parental illegal drug dependence & 231 & $0.3-4.3$ & 0.020 \\
\hline
\end{tabular}

- Crude model: adjusted for offspring gender and age at last assessment; multiple model: all covariates of interest entered into one regression model adjusted for offspring gender and maximum age as observed until the last assessment.

b All analyses including parental disorders were additionally adjusted for information level and parental age.

- Continuous variable scaled as number of years.

a Case numbers in analyses vary depending on the number of subjects with information on age at first alcohol use, age at first nicotine use and paternal or maternal alcohol or illegal drug dependence.

- AU-First: age of onset of AU in cases with AU prior to NU or no NU; NU-First: age of onset of NU in cases with NU prior to AU or no AU.

$f$ Conduct disorder, antisocial personality disorder.

8 Cannabis users with age of onset information for cannabis use; $N-142$ excluded because of unwillingness to answer drug questions truthfully.

Table

Earlier onset of alcohol and nicotine use, externalizing disorders, parental substance dependence and the risk of DSM-IV-cannabis use disorder.s

\begin{tabular}{|c|c|c|c|c|}
\hline & \multicolumn{4}{|c|}{ Cannabis use disorder $(N-294)$} \\
\hline & Hazard ratio & $95 \times \mathrm{Cl}$ & $p$ & Prob >chi ${ }^{2}$ (p-value) \\
\hline \multicolumn{5}{|l|}{ Crude model ${ }^{b}$} \\
\hline Age at first alcohol used & 0.92 & $0.8-0.9$ & 0.002 & 0.649 \\
\hline Age at first nicotine use ${ }^{d}$ & 0.97 & $0.9-1.0$ & 0.221 & 0.707 \\
\hline Externalizing disorder ${ }^{f}$ & 1.98 & $1.5-2.6$ & $<0.001$ & 0.014 \\
\hline Paternal alcohol dependence & 1.95 & $1.3-2.8$ & $<0.001$ & 0.692 \\
\hline Maternal alcohol dependence & 1.25 & $0.7-2.1$ & 0.365 & 0.225 \\
\hline Parental illegal drug dependence & 1.96 & $0.9-4.0$ & 0.065 & 0.698 \\
\hline \multicolumn{5}{|l|}{ Multiple model $=$} \\
\hline Age at first alcohol use & 0.95 & $0.8-1.0$ & 0.124 & 0.691 \\
\hline Age at first nicotine use ${ }^{d}$ & 1.04 & $0.9-1.1$ & 0.110 & 0.683 \\
\hline Age at first cannabis used & 0.77 & $0.7-0.8$ & $<0.001$ & 0.377 \\
\hline Externalizing disorder ${ }^{f}$ & 1.69 & $1.2-2.2$ & $<0.001$ & 0.014 \\
\hline Paternal alcohol dependence & 1.47 & $1.01-2.1$ & 0.042 & 0.965 \\
\hline Maternal alcohol dependence & 0.94 & $0.5-1.6$ & 0.813 & 0.354 \\
\hline Parental illegal drug dependence & 1.78 & $0.8-3.6$ & 0.110 & 0.511 \\
\hline \multicolumn{5}{|l|}{ Crude model (AU-First; NU-First)" } \\
\hline AU-First ${ }^{d}$ & 0.88 & $0.8-0.9$ & $<0.001$ & 0.927 \\
\hline NU-First ${ }^{\mathrm{d}}$ & 1.03 & $0.9-1.1$ & 0.488 & 0.213 \\
\hline \multicolumn{5}{|l|}{ Multiple model: AU-Firste } \\
\hline $\mathrm{AU}^{- \text {First }^{d}}$ & 0.94 & $0.8-1.0$ & 0.249 & 0.913 \\
\hline Age at first nicotine use $e^{d}$ & 0.99 & $0.8-1.1$ & 0.995 & 0.244 \\
\hline Age at first cannabis use $e^{d}$ & 0.72 & $0.6-0.8$ & $<0.001$ & 0.743 \\
\hline Externalizing disorder $f$ & 1.49 & $0.9-2.4$ & 0.083 & 0.483 \\
\hline Paternal alcohol dependence & 1.37 & $0.7-2.6$ & 0.325 & 0.160 \\
\hline Maternal alcohol dependence & 0.71 & $0.3-1.5$ & 0.351 & 0.112 \\
\hline \multicolumn{5}{|l|}{ Multiple model: NU-Firste } \\
\hline NU-First ${ }^{d}$ & 1.01 & $0.8-1.2$ & 0.850 & 0.522 \\
\hline Age at first alcohol use $\mathrm{d}^{\mathrm{d}}$ & 1.10 & $0.9-1.3$ & 0.240 & 0.239 \\
\hline Age at first cannabis use ${ }^{d}$ & 0.83 & $0.7-0.9$ & 0.015 & 0.594 \\
\hline Externalizing disorder ${ }^{f}$ & 1.61 & $0.9-2.8$ & 0.083 & 0.194 \\
\hline Paternal alcohol dependence & 1.62 & $0.8-3.0$ & 0.128 & 0.274 \\
\hline Maternal alcohol dependence & 2.35 & $1.09-5.0$ & 0.028 & 0.917 \\
\hline
\end{tabular}

" Schoenfeld Residual Test (test on proportional haz.ard assumption) with $p<0.05$ indicates interaction with time.

b Crude model: Stratified for offspring gender and maximum age as observed until last assessment; analyses including parental disorders were additionally adjusted for information level and parental age,

c Multiple model: all covariates entered into one regression model stratified for offspring gender and maximum age as observed until last assessment; analyses including parental disorders were additionally adjusted for information level and parental age.

Continuous variable scaled as number of years.

- AU-First: age of onset of AU in cases with AU prior to NU or no NU; NU-First: age of onset of NU in cases with NU prior to AU or no AU; in the AU-First and NU-First multiple model, parental illegal drug dependence was not included because of restricted power.

' Conduct disorder, antisocial personality disorder.

s Cannabis users with age of onset information for cannabis use; $N-142$ excluded because of unwillingness to answer drug questions truthfully. 


\section{References}

- $\quad$ Agrawal, A., Sartor, C.E., Lynskey, M.T., Grant, J.D., Pergadia, M.L., Grucza, R., Bucholz, K.K., Nelson, E.C., Madden, P.A.F., Martin, N.G., Heath, A.C., 2009. Evidence for an interaction between age at first drink and genetic influences on DSM-IV alcohol dependence symptoms. Alcoholism 33, 2047-2056.

- $\quad$ Beesdo, K., Bittner, A., Pine, D.S., Stein, M.B., Höfler, M., Lieb, R., Wittchen, H.-U., 2007. Incidence of social anxiety disorder and the consistent risk for secondary depression in the first three decades of life. Arch. Gen. Psychiatry 64, 903-912.

- $\quad$ Behrendt, S., Wittchen, H.-U., Höfler, M., Lieb, R., Beesdo, K., 2009. Transitions from first substance use to substance use disorders in adolescence: is early onset associated with a rapid escalation? Drug Alcohol Depend. 99, 68-78.

- Bonomo, Y.A., Bowes, G., Coffey, C., Carlin, J.B., Patton, G.C., 2004. Teenage drinking and the onset of alcohol dependence: a cohort study over seven years. Addiction 99, 1520-1528.

- $\quad$ Breslau, N., Fenn, N., Peterson, E.L., 1993. Early smoking initiation and nicotine dependence in a cohort of young adults. Drug Alcohol Depend. 33, 129-137.

- $\quad$ Buchmann, A.F., Schmid, B., Blomeyer, D., Becker, K., Treutlein, J., Zimmermann, U.S., JennenSteinmetz, C., Schmidt, M.H., Esser, G., Banaschewski, T., Rietschel, M., Schumann, G., Laucht, M., 2009. Impact of age at first drink on vulnerability to alcohol-related problems: testing the marker hypothesis in a prospective study of young adults. J. Psychiatr. Res. 43, 1205-1212.

- $\quad$ Caris, L., Wagner, F.A., Ríos-Bedoya, C.F., Anthony, J.C., 2009. Opportunities to use drugs and stages of drug involvement outside the United States: evidence from the Republic of Chile. Drug Alcohol Depend. 102, 30-34.

- $\quad$ Cha, Y.M., White, A.M., Kuhn, C.M., Wilson, W.A., Swartzwelder, H.S., 2006. Differential effects of delta(9)-THC on learning in adolescent and adult rats. Pharmacol. Biochem. Behav. 83, 448-455.

- Chen, C.-Y., O’Brien, M.S., Anthony, J.C., 2005. Who becomes cannabis dependent soon after onset of use? Epidemiological evidence from the United States: 2000-2001. Drug Alcohol Depend. 79, 11-22.

- Coffey, C., Carlin, J.B., Degenhardt, L., Lynskey, M., Sanci, L., Patton, G.C., 2002. Cannabis dependence in young adults: an Australian population study. Addiction 97, 187-194.

- $\quad$ Dawson, D.A., Goldstein, R.B., Chou, S.P., Ruan, W.J., Grant, B.F., 2008. Age at first drink and the first incidence of adult-onset DSM-IV alcohol use disorders. Alcohol. Clin. Exp. Res. 32, 1-12.

- $\quad$ Degenhardt, L., Chiu, W.T., Conway, K., Dierker, L., Glantz, M., Kalaydijan, A., Merikangas, K., Sampson, N., Swendsen, J., Kessler, R.C., 2009. Does the "gateway" matter?

- Associations between the order of drug use initiation and the development of drug dependence in the National Comorbidity Study Replication. Psychol. Med. 39, 157-167.

- $\quad$ DeWit, D.J., Adlaf, E.M., Offord, D.R., Ogborne, A.C., 2000. Age at first alcohol use: a risk factor for the development of alcohol disorders. Am. J. Psychiatry 157,745-750.

- $\quad$ Elkins, I.J., McGue, M., Iacono, W.G., 2007. Prospective effects of attentiondeficit/hyperactivity disorder, conduct disorder, and sex on adolescent substance use and abuse. Arch. Gen. Psychiatry 64, 1145-1152.

- $\quad$ Ellickson, P.L., Tucker, J.S., Klein, D.J., Saner, H., 2004. Antecedents and outcomes of marijuana use initiation during adolescence. Prev. Med. 39, 976-984.

- $\quad$ European Monitoring Centre for Drugs and Drug Addiction, 2010. Annual Report 2010: The State of the Drugs Problem in Europe. Publications Office of the European Union, Luxembourg.

- $\quad$ Everett, S.A., Warren, C.W., Sharp, D., Kann, L., Husten, C.G., Crosett, L.S., 1999. Initiation of cigarette smoking and subsequent smoking behavior among U.S. high school students. Prev. Med. 29, 327-333.

- $\quad$ Georgiades, K., Boyle, M.H., 2007. Adolescent tobacco and cannabis use: young adult outcomes from the Ontario Child Health Study. J. Child Psychol. Psychiatry 48,724-731.

- Grucza, R.A., Bierut, L.J., 2006. Cigarette smoking and the risk for alcohol use disorders among adolescent drinkers. Alcohol. Clin. Exp. Res. 30, 2046-2054.

- Hayatbakhsh, M.R., McGee, T.R., Bor, W., Najman, J.M., Jamrozik, K., Mamun, A.A., 2008. Child and adolescent externalizing behavior and cannabis use disorders in early adulthood: an Australian prospective birth cohort study. Addict. Behav. 33, 422-438.

- Hayatbakhsh, M.R., Najman, J.M., Jamrozik, K., Mamun, A.A., Alati, R., Bor, W., 2007. Cannabis and anxiety and depression in young adults: a large prospective study. J. Am. Acad. Child Adolesc. Psychiatry 46, 408-417.

- Kandel, D., Yamaguchi, K., 1993. From beer to crack: developmental patterns of drug involvement. Am. J. Public Health 83, 851-855. 
- $\quad$ King, S.M., Iacono, W.G., McGue, M., 2004. Childhood externalizing and internalizing psychopathology in the prediction of early substance use. Addiction 99, 1548-1559.

- $\quad$ Kokkevi, A., Gabhainn, S.N., Spyropoulou, M., HBSC, R.B.F.G.o.t., 2006. Early initiation of cannabis use: a cross-national European perspective. J. Adolesc. Health 39, 712-719.

- $\quad$ Lachner, G., Wittchen, H.-U., Perkonigg, A., Holly, A., Schuster, P., Wunderlich, U., Türk, D., Garczynski, E., Pfister, H., 1998. Structure, content and reliability of the Munich Composite International Diagnostic Interview (M-CIDI) Substance Use Sections. Eur. Addict. Res. 4, $28-41$.

- $\quad$ Lieb, R., Isensee, B., Höfler, M., Pfister, H., Wittchen, H.-U., 2001. Elterliche Alkoholbelastung und die Entwicklung von Suchtproblemen bei ihren Kindern-Ergebnisse der prospektiv-longitudinalen EDSP-Studie. Suchttherapie 2, 125-136.

- $\quad$ Lieb, R., Isensee, B., von Sydow, K., Wittchen, H.-U., 2000. The Early Developmental Stages of Psychopathology Study (EDSP): a methodological update. Eur. Addict. Res. 6, 170-182.

- $\quad$ Lieb, R., Merikangas, K., Höfler, M., Pfister, H., Isensee, B., Wittchen, H.U., 2002. Parental alcohol use disorders and alcohol use and disorders in offspring: a community study. Psychol. Med. 32, 63-78.

- $\quad$ Lynskey, M.T., Heath, A.C., Bucholz, K.K., Slutske, W.S., Madden, P.A.F., Nelson, E.C., Statham, D.J., Martin, N.G., 2003. Escalation of drug use in early-onset cannabis users vs co-twin controls. JAMA 289, 427-433.

- Malone, D.T., Hill, M.N., Rubino, T., 2010. Adolescent cannabis use and psychosis: epidemiology and neurodevelopmental models. Br. J. Pharmacol. 160, 511-522.

- $\quad$ McGue, M., Iacono, W.G., 2008. The adolescent origins of substance use disorders. Int. J. Methods Psychiatr. Res. 17 (S1), 30-38.

- $\quad$ Merikangas, K.R., Stevens, D.E., Fenton, B., Stolar, M., O’Malley, S., Woods, S.W., Risch, N., 1998. Co-morbidity and familial aggregation of alcoholism and anxiety disorders. Psychol. Med. 28, 773-788.

- $\quad$ Monshouwer, K., Smit, F., de Graaf, R., van Os, J., Vollebergh, W., 2005. First cannabis use: does onset shift to younger ages? Findings from 1988 to 2003 from the Dutch National School Survey on Substance Use. Addiction 100, 963-970.

- $\quad$ Morral, A.R., McCaffrey, D.F., Paddock, S.M., 2002. Reassessing the marijuana gateway effect. Addiction 97, 1493-1504.

- Nixon, K., McClain, J.A., 2010. Adolescence as a critical window for developing an alcohol use disorder: current findings in neuroscience. Curr. Opin. Psychiatr. 23, 227-232.

- $\quad$ O’Shea, M., Singh, M.E., McGregor, I.S., Mallet, P.E., 2004. Chronic cannabinoid exposure produces lasting memory impairment and increased anxiety in adolescent but not adult rats. J. Psychopharmacol. $18,502-508$.

- $\quad$ Obot, I.S., Wagner, F.A., Anthony, J.C., 2001. Early onset and recent drug use among children of parents with alcohol problems: data from a national epidemiologic survey. Drug Alcohol Depend. 65, $1-8$.

- $\quad$ Palmer, R.H.C., Young, S.E., Hopfer, C.J., Corley, R.P., Stallings, M.C., Crowley, T.J., Hewitt, J.K., 2009. Developmental epidemiology of drug use and abuse in adolescence and young adulthood: evidence of generalized risk. Drug Alcohol Depend. 102, 78-87.

- $\quad$ Pape, H., Rossow, I., Storvoll, E.E., 2009. Under double influence: assessment of imultaneous alcohol and cannabis use in general youth populations. Drug Alcohol Depend. 101, 69-73.

- $\quad$ Patton, G.C., Coffey, C., Carlin, J.B., Degenhardt, L., Lynskey, M., Hall, W., 2002. Cannabis use and mental health in young people: cohort study. Br. Med. J. 325, 1195-1198.

- $\quad$ Perkins, K.A., Coddington, S.B., Karelitz, J.B., Jetton, C., Scott, J.A., Wilson, A.S., Lerman, C., 2009. Variability in initial nicotine sensitivity due to sex, history of drug use, and parental smoking. Drug Alcohol Depend. 99, 47-57.

- $\quad$ Poelen, E.A.P., Scholte, R.H.J., Engels, R.C.M.E., Boomsma, D.I., Willemsen, G., 2005. Prevalence and trends of alcohol use and misuse among adolescents and young adults in the Netherlands from 1993 to 2000. Drug Alcohol Depend. 79, 413-421.

- Quinn, H.R., Matsumoto, I., Callaghan, P.D., Long, L.E., Arnold, J.C., Gunasekaran, N., Thompson, M.R., Dawson, B., Mallet, P.E., Kashem, M.A., Matsuda-Matsumoto, H., Iwazaki, T., McGregor, I.S., 2008. Adolescent rats find repeated Delta(9)-THC less aversive than adult rats but display greater residual cognitive deficits and changes in hippocampal protein expression following exposure. Neuropsychopharmacology 33, 1113-1126.

- $\quad$ Reed, V., Gander, F., Pfister, H., Steiger, A., Sonntag, H., Trenkwalder, C., Sonntag, A., Hundt, W., Wittchen, H.-U., 1998. To what degree does the Composite International Diagnostic Interview (CIDI) correctly identify DSM-IV disorders? Testing validity issues in a clinical sample. Int. J. Methods Psychiatr. Res. 7, 142-155.

- $\quad$ Royall, R.M., 1986. Model robust confidence intervals using maximum likelihood estimators. Stat. Rev. 54, 221-226. 
- Sartor, C.E., Agrawal, A., Lynskey, M.T., Bucholz, K.K., Madden, P.A.F., Heath, A.C., 2009. Common genetic influences on the timing of first use for alcohol, cigarettes, and cannabis in young AfricanAmerican women. Drug Alcohol Depend. 102, 49-55.

- Schramm-Sapyta, N.L., Cha, Y.M., Chaudry, S., Wilson, W.A., Schwartzwelder, H.S., Kuhn, C.M., 2007. Differential anxiogenic, aversive, and locomotor effects of THC in adolescent and adult rats. Psychpharmacology 191, 867-877.

- Schramm-Sapyta, N.L., Walker, Q.D., Caster, J.M., Levin, E.D., Kuhn, C.M., 2009. Are adolescents more vulnerable to drug addiction than adults? Evidence from animal models. Psychopharmacology 206, 1-21.

- StataCorp (Ed.), 2009. Stata Statistical Software: Release 11. 0. StataCorp LP, College Station, TX.

- Therneau, T., Grambsch, P., 2000. Modeling Survival Data: Extending the Cox Model. Springer, New York.

- United Nations Office on Drugs and Crime, 2010. World Drug Report 2010. United Nations, New York.

- Vandeleur, C.L., Rothen, S., Jeanpretre, N., Lustenberger, Y., Gamma, F., Ayer, E., Ferrero, F., Fleischmann, A., Besson, J., Sisbane, F., Preisig, M., 2008. Inter-informant agreement and prevalence estimates for substance use disorders: direct interview versus family history method. Drug Alcohol Depend., 92.

- $\quad$ von Sydow, K., Lieb, R., Pfister, H., Höfler, M., Wittchen, H.-U., 2002. What predicts incident use of cannabis and progression to abuse or dependence? A 4-year prospective examination of risk factors in a community sample of adolescents and young adults. Drug Alcohol Depend. 68, 49-64.

- Wagner, F.A., Anthony, J.C., 2002a. From first drug use to drug dependence: developmental periods of risk for dependence upon marijuana, cocaine, and alcohol. Neuropsychopharmacology 26, 479-488.

- Wagner, F.A., Anthony, J.C., 2002b. Into the world of illegal drugs: exposure opportunity and other mechanisms linking the use of alcohol, tobacco, marijuana, and cocaine. Am. J. Epidemiol. 155, 918 925.

- $\quad$ Wittchen, H.-U., Behrendt, S., Höfler, M., Perkonigg, A., Lieb, R., Bühringer, G., Beesdo, K., 2008. What are the high risk periods for incident substance use and transitions to abuse and dependence? Implications for early intervention and prevention. Int. J. Methods Psychiatr. Res. 17, 16-29.

- Wittchen, H.-U., Lachner, G., Wunderlich, U., Pfister, H., 1998a. Test-retest reliability of the computerized DSM-IV version of the Munich-Composite International Diagnostic Interview (M-CIDI). Soc. Psychiatry Psychiatr. Epidemiol. 33, 568-578.

- Wittchen, H.-U., Perkonigg, A., Lachner, G., Nelson, C.B., 1998b. Early Developmental Stages of Psychopathology Study (EDSP): objectives and design. Eur. Addict. Res. 4, 18-27.

- Wittchen, H.-U., Pfister, H., 1997. DIA-X Interview. Instruktionsmanual zur Durchführung von DIA$\mathrm{X}$-Interviews. Swets and Zeitlinger, Frankfurt.

- Wittchen, H.-U., Semmler, G., 1990. Composite International Diagnostic Interview (CIDI). Beltz, Weinheim.

- Young, S.E., Corley, R.P., Stallings, M.C., Rhee, T.J., Crowley, T.J., Hewitt, J.K., 2002. Substance use, abuse and dependence in adolescence: prevalence, symptom profiles and correlates. Drug Alcohol Depend. 68, 309-322. 